\title{
ON DIFFERENTIAL EQUATIONS FOR ORTHOGONAL POLYNOMIALS
}

\author{
Mourad E. H. Ismail and Jet Wimp
}

\begin{abstract}
We find four linear independent solutions of the fourth-order differential equation satisfied by the associated Jacobi polynomials. We show that the coefficients in the lowering operator for general orthogonal polynomials satisfy inhomogeneous four-term recurrence relations and derive further properties of them. In addition, we show that the associated polynomials $\left\{p_{n}^{(c)}(x)\right\}$ for positive integers $c$ satisfy a linear differential equation of order four, we identify a basis of solutions of the differential equation, and we establish similar results for co-recursive polynomials.
\end{abstract}

\section{Introduction}

Let $\left\{p_{n}(x)\right\}$ be orthonormal with respect to a weight function $w$ supported on an interval $[a, b]$, finite or infinite, that is

$$
\int_{a}^{b} p_{m}(x) p_{n}(x) w(x) d x=\delta_{m, n}
$$

With the weight function $w$ we associate an external field $v$ through

$$
w(x)=e^{-v(x)}
$$

The $p_{n}$ s satisfy a three-term recurrence relation

$$
x p_{n}(x)=a_{n+1} p_{n+1}(x)+b_{n} p_{n}(x)+a_{n} p_{n-1}(x), \quad n>0,
$$

with

$$
p_{0}(x)=1, \quad p_{1}(x)=\left(x-b_{0}\right) / a_{1} .
$$

We shall assume

$$
y^{n} \frac{v^{\prime}(x)-v^{\prime}(y)}{x-y} w(y), \quad n=0,1, \ldots
$$

Received January 5, 1998, revised September 23, 1998.

1991 Mathematics Subject Classification: Primary 42C05, secondary 33C45.

Key words and phrases: orthogonal polynomials, functions of the second kind, recurrence relations, differential equations, associated polynomials, co-recursive polynomials. 
are integrable functions of $y$ on $[a, b]$ for every fixed $x$ in $[a, b]$. Define $A_{n}$ and $B_{n}$ by

$$
\begin{aligned}
A_{n}(x)= & \frac{a_{n} w\left(b^{-}\right) p_{n}^{2}\left(b^{-}\right)}{b-x}+\frac{a_{n} w\left(a^{+}\right) p_{n}^{2}\left(a^{+}\right)}{x-a} \\
& \quad+a_{n} \int_{a}^{b} \frac{v^{\prime}(x)-v^{\prime}(y)}{x-y} p_{n}^{2}(y) w(y) d y \\
B_{n}(x)= & \frac{a_{n} w\left(a^{+}\right) p_{n}\left(a^{+}\right) p_{n-1}\left(a^{+}\right)}{x-a}+\frac{a_{n} w\left(b^{-}\right) p_{n}\left(b^{-}\right) p_{n-1}\left(b^{-}\right)}{b-x} \\
& +a_{n} \int_{a}^{b} \frac{v^{\prime}(x)-v^{\prime}(y)}{x-y} p_{n}(y) p_{n-1}(y) w(y) d y .
\end{aligned}
$$

The boundary terms in (1.6) and (1.7) are assumed to exist. It was proved in [5] that the orthonormal polynomials $\left\{p_{n}(x)\right\}$ satisfy the differential equation

$$
p_{n}^{\prime \prime}(x)+R_{n}(x) p_{n}^{\prime}(x)+S_{n}(x) p_{n}(x)=0
$$

where

$$
\begin{aligned}
& R_{n}(x):=- {\left[v^{\prime}(x)+\frac{A_{n}^{\prime}(x)}{A_{n}(x)}\right] } \\
& S_{n}(x):=B_{n}^{\prime}(x)-B_{n}(x) \frac{A_{n}^{\prime}(x)}{A_{n}(x)}-B_{n}(x)\left[v^{\prime}(x)+B_{n}(x)\right] \\
& \quad+\frac{a_{n}}{a_{n-1}} A_{n}(x) A_{n-1}(x) .
\end{aligned}
$$

Earlier versions are in [3, 4]. The differential equation (1.8) is proved in [5] by first proving the lowering relation

$$
p_{n}^{\prime}(x)=A_{n}(x) p_{n-1}(x)-B_{n}(x) p_{n}(x)
$$

then using the three-term recurrence relation (1.3) to eliminate $p_{n-1}(x)$. The differential recurrence relation (1.11) is of independent interest and will be used in Sections 4 and 5 .

In this work, we first study properties of the sequences $A_{n}(z)$ and $B_{n}(z)$. In Section 2 , we shall prove the following theorem.

Theorem 1.1. The $A_{n} s$ and $B_{n} s$ satisfy

$$
\begin{aligned}
& B_{n+1}(x)+B_{n}(x)=\frac{x-b_{n}}{a_{n}} A_{n}(x)-v^{\prime}(x), \\
& B_{n+1}(x)-B_{n}(x)=\frac{a_{n+1} A_{n+1}(x)}{x-b_{n}}-\frac{a_{n}^{2} A_{n-1}(x)}{a_{n-1}\left(x-b_{n}\right)}-\frac{1}{x-b_{n}} .
\end{aligned}
$$

In Section 2, we will also indicate some of the consequences of Theorem 1.1, such as showing that the $A_{n}$ s and $B_{n}$ s satisfy non-homogeneous third-order difference equations in $n$. In Section 2, we also give a finite sum representation for $F_{n}(x)$, as defined by

$$
F_{n}(x):=\frac{a_{n}}{a_{n-1}} A_{n}(x) A_{n-1}(x)-B_{n}(x)\left[v^{\prime}(x)+B_{n}(x)\right] .
$$

This representation of $F_{n}(x)$ is in (2.12) and is useful, for example, when $v^{\prime}$ is a polynomial because it gives the correct degree for the above expression, hence the correct degree for $A_{n}(x) S_{n}(x)$ in (1.8). We also prove an integral representation for $F_{n}(x)$ when $w\left(a^{+}\right)=w\left(b^{-}\right)=0$; see $(2.14)$. 
Ismail [10] proved that the numerator polynomials of the $p_{n} \mathrm{~s}$ generated by (1.3) and (1.4) satisfy a fourth-order differential equation. He also gave three linearly independent solutions of this differential equation. Earlier, Wimp [21] found a linear fourth-order differential equation satisfied by the associated Jacobi polynomials. In Section 3, we give a basis of solutions of Wimp's differential equation. This seems to be the first instance of an explicit basis of solutions made of non-elementary transcendental functions for a differential equation of such high order with such complex structure. Observe that the numerator polynomials are associated polynomials with the association parameter equal to unity. It is hoped that the result for the special equation of associated Jacobi polynomials will shed some light on the general structure of differential equations for general associated polynomials.

In Section 4, we establish a linear fourth-order differential equation for the associated polynomials of the orthonormal polynomials generated by (1.3) and (1.4) when the association parameter is at least one. This extends a result in [10]. We also provide a basis of solutions of the fourth-order differential equation formed by taking products of the polynomials and/or functions of the second kind. Finding a basis of solutions of such a fourth-order differential equation in this generality is a surprise. Although the associated polynomials may satisfy a linear second-order differential equation as predicted by (1.8)-(1.10), the weight functions for the associated polynomials may be too complicated to give reasonable expressions for their $A_{n} \mathrm{~s}$ and $B_{n} \mathrm{~s}$. For samples of the weight functions for associated orthogonal polynomials, we refer the interested reader to the recent works $[21,12,13]$. In Section 5 , we show that the co-recursive polynomials [6] satisfy fourth-order differential equations.

This paper is part of a series of papers on the subject of functional equations satisfied by orthogonal polynomials. Finite difference and $q$-analogues of this work are in preparation, including $q$-analogues of discriminants of polynomials.

\section{Recurrence relations}

In this section, we study properties of $A_{n}(x)$ and $B_{n}(x)$. Before proving Theorem 1.1, we note that formula (1.12) is in [5] when $w$ vanishes at the end points $a$ and $b$. It is interesting to observe that although the boundary values at $a$ and $b$ appear in $A_{n}(x)$ and $B_{n}(x)$, they do not appear in the coefficients in the identities (1.12) and (1.13).

Proof of Theorem 1.1. It follows from (1.7) that the left-hand side of (1.12) is the sum of four boundary terms and two integrals. In view of the relations (1.3) and (1.4), the boundary terms at $a^{+}$combine to give

$$
\frac{a-b_{n}}{x-a} w\left(a^{+}\right) p_{n}^{2}\left(a^{+}\right)
$$

which is

$$
\frac{x-b_{n}}{x-a} w\left(a^{+}\right) p_{n}^{2}\left(a^{+}\right)-w\left(a^{+}\right) p_{n}^{2}\left(a^{+}\right) .
$$

Similar results hold for boundary terms involving $b^{-}$. Thus the boundary terms on the left-hand side of (1.12) exceed the boundary terms on its right-hand side by $E_{n}$, say,

$$
E_{n}=w\left(b^{-}\right) p_{n}^{2}\left(b^{-}\right)-w\left(a^{+}\right) p_{n}^{2}\left(a^{+}\right)
$$


The difference between the terms involving integrals on the left-hand side of (1.12) and the right-hand side may be combined by using (1.3) to become

$$
\int_{a}^{b} \frac{v^{\prime}(x)-v^{\prime}(y)}{x-y}(y-x) p_{n}^{2}(y) w(y) d y
$$

which is

$$
-v^{\prime}(x)-\int_{a}^{b} p_{n}^{2}(y) w^{\prime}(y) d y
$$

On the other hand, integration by parts gives

$$
\int_{a}^{b} p_{n}^{2}(y)\left(-v^{\prime}(y)\right) w(y) d y=\int_{a}^{b} p_{n}^{2}(y) w^{\prime}(y) d y=0,
$$

due to the orthogonality of the $p_{n} \mathrm{~s}$. This proves (1.12). To prove (1.13), we set

$$
a_{n+1} A_{n+1}(x)-\frac{a_{n}^{2} A_{n-1}(x)}{a_{n-1}}=I+B T,
$$

where $I$ and $B T$ stand for integrals and boundary terms in the expression on the left-hand side of (2.3). Now the three-term recurrence relation (1.3) gives

$$
\begin{aligned}
I= & \int_{a}^{b} \frac{v^{\prime}(x)-v^{\prime}(y)}{x-y}\left[a_{n+1} p_{n+1}(y)-a_{n} p_{n-1}(y)\right]\left(y-b_{n}\right) p_{n}(y) w(y) d y \\
=\left(x-b_{n}\right)\left[B_{n+1}(x)-B_{n}(x)\right] & \\
& +\left(x-b_{n}\right)\left[\frac{w\left(a^{+}\right) p_{n}\left(a^{+}\right)}{x-a}\left\{a_{n} p_{n-1}\left(a^{+}\right)-a_{n+1} p_{n+1}\left(a^{+}\right)\right\}\right. \\
& \left.+\frac{w\left(b^{-}\right) p_{n}\left(b^{-}\right)}{b-x}\left\{a_{n} p_{n-1}\left(b^{-}\right)-a_{n+1} p_{n+1}\left(b^{-}\right)\right\}\right] \\
& -\int_{a}^{b}\left[v^{\prime}(x)-v^{\prime}(y)\right]\left[a_{n+1} p_{n+1}(y)-a_{n} p_{n-1}(y)\right] p_{n}(y) w(y) d y .
\end{aligned}
$$

The remaining integral in $I$ can be evaluated as follows:

$$
\begin{aligned}
\int_{a}^{b}\left[v^{\prime}(x)-v^{\prime}(y)\right]\left[a_{n+1} p_{n+1}(y)-a_{n} p_{n-1}(y)\right] p_{n}(y) w(y) d y \\
=\int_{a}^{b}\left[a_{n+1} p_{n+1}(y)-a_{n} p_{n-1}(y)\right] p_{n}(y) w^{\prime}(y) d y \\
=\left.\left\{a_{n+1} p_{n+1}(y)-a_{n} p_{n-1}(y)\right\} w(y) p_{n}(y)\right|_{y=a} ^{b} \\
\quad-\int_{a}^{b}\left[a_{n+1} p_{n}(y) p_{n+1}^{\prime}(y)-a_{n} p_{n-1}(y) p_{n}^{\prime}(y)\right] w(y) d y
\end{aligned}
$$

Since the coefficient of $x^{n}$ in $p_{n}(x)$ is $\left(a_{1} \ldots a_{n}\right)^{-1}$, then

$$
\int_{a}^{b} a_{n+1} p_{n}(y) p_{n+1}^{\prime}(y) w(y) d y=(n+1) \int_{a}^{b} p_{n}^{2}(y) w(y) d y=n+1
$$


and after putting all this information together, we establish

$$
\begin{gathered}
I=\left(x-b_{n}\right)\left[B_{n+1}(x)-B_{n}(x)\right]-\left.\left\{a_{n+1} p_{n+1}(y)-a_{n} p_{n-1}(y)\right\} w(w) p_{n}(y)\right|_{a} ^{b} \\
+\left(x-b_{n}\right)\left[\frac{w\left(a^{+}\right) p_{n}\left(a^{+}\right)}{x-a}\left\{a_{n} p_{n-1}\left(a^{+}\right)-a_{n+1} p_{n+1}\left(a^{+}\right)\right\}\right. \\
\left.+\frac{w\left(b^{-}\right) p_{n}\left(b^{-}\right)}{b-x}\left\{a_{n} p_{n-1}\left(b^{-}\right)-a_{n+1} p_{n+1}\left(b^{-}\right)\right\}\right]-1 .
\end{gathered}
$$

Now the boundary terms above combine, and when compared with the terms $B T$ in (2.3), we find

$$
I+B T=\left(x-b_{n}\right)\left[B_{n+1}(x)-B_{n}(x)\right]
$$

which completes the proof of (1.13) and Theorem 1.1.

Once we have established (1.12) and (1.13), it immediately follows that

$$
2 B_{n+1}(x)=\frac{x-b_{n}}{a_{n}} A_{n}(x)+\frac{a_{n+1} A_{n+1}(x)}{x-b_{n}}-\frac{a_{n}^{2} A_{n-1}(x)}{a_{n-1}\left(x-b_{n}\right)}-v^{\prime}(x)-\frac{1}{x-b_{n}}
$$

and

$$
2 B_{n}(x)=\frac{x-b_{n}}{a_{n}} A_{n}(x)-\frac{a_{n+1} A_{n+1}(x)}{x-b_{n}}+\frac{a_{n}^{2} A_{n-1}(x)}{a_{n-1}\left(x-b_{n}\right)}-v^{\prime}(x)+\frac{1}{x-b_{n}} .
$$

For consistency, it follows that $A_{n}$ must satisfy the inhomogeneous four-term recurrence relation

$$
\begin{aligned}
\frac{a_{n+2} A_{n+2}(x)}{x-b_{n+1}}= & {\left[\frac{x-b_{n+1}}{a_{n+1}}-\frac{a_{n+1}}{x-b_{n}}\right] A_{n+1}(x)+\left[\frac{a_{n+1}^{2}}{a_{n}\left(x-b_{n+1}\right)}-\frac{\left(x-b_{n}\right)}{a_{n}}\right] A_{n}(x) } \\
& +\frac{a_{n}^{2}}{a_{n-1}\left(x-b_{n}\right)} A_{n-1}(x)+\frac{1}{x-b_{n}}+\frac{1}{x-b_{n+1}}, \quad n>1 . \quad(2.9)
\end{aligned}
$$

We can extend the validity of (2.9) to the cases $n=0$ and $n=1$ if we adopt the convention

$$
\begin{gathered}
a_{0}:=1, \quad p_{-1}:=0 \\
\frac{A_{0}(x)}{a_{0}}:=\frac{w\left(a^{+}\right)}{x-a}+\frac{w\left(b^{-}\right)}{b-x}+\int_{a}^{b} \frac{v^{\prime}(x)-v^{\prime}(y)}{x-y} w(y) d y .
\end{gathered}
$$

Thus,

$$
B_{0}=A_{-1}(x)=0 \text {. }
$$

We next eliminate $A_{n}(x)$ from (1.12) and (1.13) and, after some simple algebra, we find that the $B_{n}$ s also satisfy the inhomogeneous four-term recurrence relation

$$
\begin{aligned}
B_{n+2}(x)=[ & \left.\frac{\left(x-b_{n}\right)\left(x-b_{n+1}\right)}{a_{n+1}^{2}}-1\right] B_{n+1}(x) \\
& +\left[\frac{a_{n}^{2}\left(x-b_{n+1}\right)}{a_{n+1}^{2}\left(x-b_{n-1}\right)}-\frac{\left(x-b_{n}\right)\left(x-b_{n+1}\right)}{a_{n+1}^{2}}\right] B_{n}(x) \\
& +\frac{a_{n}^{2}\left(x-b_{n+1}\right)}{a_{n+1}^{2}\left(x-b_{n-1}\right)} B_{n-1}(x)+\frac{\left(x-b_{n+1}\right)}{a_{n+1}^{2}} \\
& +\left[\frac{a_{n}^{2}\left(x-b_{n+1}\right)}{a_{n+1}^{2}\left(x-b_{n-1}\right)}-1\right] v^{\prime}(x), \quad n>1 .
\end{aligned}
$$


Theorem 2.1. For all $n \geq 0$, the functions $A_{n}(x)$ and $B_{n}(x)$ are linear combinations of $A_{0}(x)$ and $v^{\prime}(x)$ with rational function coefficients.

Proof. The statement can be readily verified for $n=0,1$. The theorem then follows by induction from the recurrence relations (2.9) and (2.11).

Our next result is an alternate representation of $F_{n}(x)$ defined in (1.14).

Theorem 2.2. We have

$$
F_{n}(x)=\sum_{k=0}^{n-1} A_{k}(x) / a_{k}
$$

Proof. Express $F_{n+1}(x)-F_{n}(x)$ as

$$
\begin{aligned}
\frac{a_{n+1}}{a_{n}} A_{n}(x) A_{n+1}(x)-\frac{a_{n}}{a_{n-1}} A_{n}(x) A_{n-1}(x) & \\
+ & {\left[B_{n}(x)-B_{n+1}(x)\right]\left[B_{n}(x)+B_{n+1}(x)+v^{\prime}(x)\right] . }
\end{aligned}
$$

Now eliminate $B_{n}(x)$ using (1.12) and (1.13) to see that the above expression gives

$$
\begin{aligned}
F_{n+1}(x)-F_{n}(x)= & {\left[\frac{a_{n+1}}{a_{n}} A_{n+1}(x)-\frac{a_{n}}{a_{n-1}} A_{n-1}(x)\right] A_{n}(x) } \\
& +\frac{x-b_{n}}{a_{n}} A_{n}(x)\left[\frac{1}{x-b_{n}}+\frac{a_{n}^{2} A_{n-1}(x)}{a_{n-1}\left(x-b_{n}\right)}-\frac{a_{n+1} A_{n+1}(x)}{x-b_{n}}\right],
\end{aligned}
$$

which simplifies to $A_{n}(x) / a_{n}$ when $n>0$. When $n=0$, the relationship (2.13) can be verified directly with $F_{0}(x):=0$. This gives $F_{n}(x)$ as a telescoping sum and the theorem follows.

Theorem 2.3. If $w\left(a^{+}\right)=w\left(b^{-}\right)=0$, then $F_{n}(x)$ has the integral representation

$$
F_{n}(x)=a_{n}^{2} \int_{a}^{b} \int_{a}^{b} \frac{v^{\prime}(x)-v^{\prime}(y)}{x-y} \frac{v^{\prime}(t) w(y) w(t)}{x-t}\left[p_{n}(t) p_{n-1}(y)-p_{n}(y) p_{n-1}(t)\right]^{2} d y d t
$$

Proof. Formulas (1.6) and (1.7) yield

$$
\begin{aligned}
F_{n}(x)+v^{\prime}(x) B_{n}(x)= & a_{n} \int_{a}^{b} \int_{a}^{b} \frac{v^{\prime}(x)-v^{\prime}(y)}{x-y} \frac{v^{\prime}(x)-v^{\prime}(t)}{x-t} p_{n}(t) p_{n-1}(y) \\
& \times a_{n}\left[p_{n}(t) p_{n-1}(y)-p_{n}(y) p_{n-1}(t)\right] w(y) w(t) d y d t .
\end{aligned}
$$

Using the Christoffel-Darboux formula

$$
a_{n}\left[p_{n}(t) p_{n-1}(y)-p_{n}(y) p_{n-1}(t)\right]=(t-y) \sum_{k=0}^{n-1} p_{k}(y) p_{k}(t)
$$

and the partial fraction decomposition

$$
\frac{t-y}{(x-y)(x-t)}=\frac{1}{x-t}-\frac{1}{x-y}
$$


we arrive at

$$
\begin{aligned}
& F_{n}(x)+v^{\prime}(x) B_{n}(x) \\
& \quad=a_{n} \int_{a}^{b} \frac{v^{\prime}(x)-v^{\prime}(y)}{x-y} p_{n-1}(y) \int_{a}^{b} v^{\prime}(t) w(t) p_{n}(t) \sum_{k=0}^{n-1} p_{k}(y) p_{k}(t) d t d y \\
& =\int_{a}^{b} \int_{a}^{b} \frac{v^{\prime}(x)-v^{\prime}(t)}{x-t}\left[v^{\prime}(x)-v^{\prime}(y)\right] p_{n}(t) p_{n-1}(y) \sum_{k=0}^{n-1} p_{k}(y) p_{k}(t) d t d y \\
& =v^{\prime}(x) a_{n} \int_{a}^{b} \frac{v^{\prime}(x)-v^{\prime}(t)}{x-t} p_{n}(t) p_{n-1}(t)+\text { the right-hand side of }(2.14)
\end{aligned}
$$

Corollary 2.4. $F_{N}(x)$ is a polynomial of degree $2 m-2$ when $v$ is a polynomial of degree $2 m$.

Proof. It is easy to see from (1.6) and (1.7) that $A_{n}(x) / a_{n}$ has degree $2 m-2$ with leading term $2 m x^{2 m-2}$. Hence, $(2.12)$ shows that $F_{n}(x)$ has degree $2 m-2$ with leading term $2 m n x^{2 m-2}$.

\section{Some classical associated polynomials}

In this section, we explore some of the properties of the differential equation satisfied by the associated Jacobi polynomials, $R_{n}^{(\alpha, \beta)}(x ; c)$. Recall the recurrence for the associated polynomials is formed from that for the classical Jacobi polynomials simply by replacing $n$ wherever it appears by $n+c$. It can be verified directly, using Sister Celine's method [17] that two linearly independent solutions of the recurrence are

$$
\begin{aligned}
u_{n}= & \widehat{P}_{n+c}^{(\alpha, \beta)}(x)=\left(\begin{array}{c}
\alpha+n+c \\
n+c
\end{array}\right){ }_{2} F_{1}\left(\begin{array}{c|c}
-n-c, n+\gamma+c \\
\alpha+1
\end{array} \mid 1-x\right) \\
v_{n}= & \widehat{Q}_{n+c}^{(\alpha, \beta)}(x)=-\frac{\pi}{2} \csc (\alpha \pi) \widehat{P}_{n+c}^{(\alpha, \beta)}(x)+\frac{\cos (\alpha \pi) \Gamma(\alpha) \Gamma(n+\beta+c+1)}{2 \Gamma(n+\gamma+c)} \\
& \times(1-x)^{-\alpha} x^{-\beta}{ }_{2} F_{1}\left(\begin{array}{c}
n+c+1,1-n-c-\gamma \\
1-\alpha
\end{array} \mid 1-x\right), \quad 0<x<1 .
\end{aligned}
$$

Note that when $c$ is an integer, the first and second function above are just the traditional Jacobi polynomial and the Jacobi function of the second kind, respectively, shifted to the interval $0<x<1$.

By the theory of linear difference equations, $R_{n}^{(\alpha, \beta)}(x ; c)$ must be a linear combination of these two functions

$$
R_{n}^{(\alpha, \beta)}(x ; c)=A \widehat{P}_{n+c}^{(\alpha, \beta)}(x)+B \widehat{Q}_{n+c}^{(\alpha, \beta)}(x) .
$$

Solving for $A, B$ from the conditions at $n=-1$ and $n=0$ gives

$$
\begin{gathered}
R_{n}^{(\alpha, \beta)}(x ; c)=\left[\widehat{Q}_{c-1}^{(\alpha, \beta)}(x) \widehat{P}_{n+c}^{(\alpha, \beta)}(x)-\widehat{P}_{c-1}^{(\alpha, \beta)}(x) \widehat{Q}_{n+c}^{(\alpha, \beta)}(x)\right] / \Delta \\
\Delta=\widehat{Q}_{c-1}^{(\alpha, \beta)}(x) \widehat{P}_{c}^{(\alpha, \beta)}(x)-\widehat{P}_{c-1}^{(\alpha, \beta)}(x) \widehat{Q}_{c}^{(\alpha, \beta)}(x) .
\end{gathered}
$$


Using a formula in [7, Vol. 1, p. 172, formula (26) adjusted to the interval $0<x<1]$, we find that

$$
\Delta=D(1-x)^{-\alpha} x^{-\beta}, \quad D=\frac{\cos (\alpha \pi) \Gamma(\alpha+c) \Gamma(\beta+c)(\gamma+2 c-1)}{2 \Gamma(c+1) \Gamma(\gamma+c)} .
$$

We write $R_{n}^{(\alpha, \beta)}(x ; c)$ as

$$
\begin{gathered}
R_{n}^{(\alpha, \beta)}(x ; c)=\frac{1}{D}\left(s_{1} t_{1}-s_{2} t_{2}\right), \\
s_{1}=(1-x)^{\alpha} x^{\beta} \widehat{Q}_{c-1}^{(\alpha, \beta)}(x), \quad s_{2}=(1-x)^{\alpha} x^{\beta} \widehat{P}_{c-1}^{(\alpha, \beta)}(x), \\
t_{1}=\widehat{P}_{n+c}^{(\alpha, \beta)}(x), \quad t_{2}=\widehat{Q}_{n+c}^{(\alpha, \beta)}(x),
\end{gathered}
$$

where $s_{1}, s_{2}$ satisfy the same second-order homogeneous linear homogeneous equation, and $t_{1}, t_{2}$ satisfy the same second-order homogeneous differential equation. These equations can be found from those for the relevant Gaussian hypergeometric functions. Now, if $u$ and $v$ satisfy linear homogeneous differential equations, their product $W=$ $u v$ will satisfy a fourth-order homogeneous linear differential equation. To find it, start with the equation $W=u v$, differentiate this equation, differentiate again, eliminating $u$ and $v$, and differentiate again, eliminating $u$ and $v$. The five resulting equations for $W, W^{\prime}, W^{\prime \prime}, W^{\prime \prime \prime}$, and $W^{\prime \prime \prime \prime}$ involve the four "unknown" quantities $u v, u^{\prime} v, u v^{\prime}$, and $u^{\prime} v^{\prime}$. Construct the eliminant for these equations and the result is the desired fourthorder equation. This procedure is horrendous in practice and, before computer algebra systems, was usually unworkable. However, with the advent of MAPLE, Mathematica, etc., it now is fairly easy to accomplish. In the paper [21], the following fourth-order linear homogeneous differential equation for $R_{n}^{(\alpha, \beta)}(x ; c)$ was given:

$$
A_{0} y^{\prime \prime \prime \prime}+A_{1} y^{\prime \prime \prime}+A_{2} y^{\prime \prime}+A_{4} y=0
$$

where

$$
\begin{aligned}
A_{0}= & (x-1)^{2} x^{2}, \quad A_{1}=5 x(x-1)(2 x-1), \\
A_{2}= & -\left[x^{2}\left(2 K+2 C+\gamma^{2}-25\right)\right. \\
& \left.+x(-2 K-2 C-2 \beta \gamma-2 \gamma+2 \beta+26)+\beta^{2}-4\right], \\
A_{3}= & -3\left[x\left(2 K+2 C+\gamma^{2}-5\right)-K-C-\gamma \beta-\gamma+\beta+3\right], \\
A_{4}= & n(n+2)(n+2 c+\gamma)(n+2 c+\gamma-2),
\end{aligned}
$$

and

$$
K=(n+c)(n+\gamma+c), \quad C=(c-1)(\gamma+c-1) .
$$

Because of the distinct asymptotic behavior of the functions

$$
t_{1}=\widehat{P}_{n+c}^{(\alpha, \beta)}(x), \quad t_{2}=\widehat{Q}_{n+c}^{(\alpha, \beta)}(x),
$$

all four functions

$$
s_{i} t_{j}, \quad i, j=1,2,
$$

are linearly independent. Thus, they constitute a basis of solutions of the differential equation (3.10). The case $c=1$ is particularly interesting. Then we obtain a basis of solutions for the differential equation satisfied by the numerator polynomials in the 
$[n / n+1]$ Padé approximant to the ratio of two Gaussian hypergeometric functions (see the reference [21] for details):

$$
\frac{R_{n}^{(\alpha, \beta)}(x ; 1)}{\widehat{P}_{n+1}^{(\alpha, \beta)}(x)}
$$

A basis of solutions of the differential equation satisfied by $R_{n}^{(\alpha, \beta)}(x ; 1)$ is

$$
\begin{aligned}
(1-x)^{\alpha} x^{\beta} \widehat{P}_{n+1}^{(\alpha, \beta)}(x), & (1-x)^{\alpha} x^{\beta} \widehat{Q}_{n+1}^{(\alpha, \beta)}(x), \\
(1-x)^{\alpha} x^{\beta} \widehat{Q}_{0}^{(\alpha, \beta)}(x) \widehat{P}_{n+1}^{(\alpha, \beta)}(x), & (1-x)^{\alpha} x^{\beta} \widehat{Q}_{0}^{(\alpha, \beta)}(x) \widehat{Q}_{n+1}^{(\alpha, \beta)}(x) .
\end{aligned}
$$

Replacing $x$ by $1-x / \beta$ in the equation (3.10) and letting $\beta \rightarrow \infty$ produces the fourthorder differential equation satisfied by the associated Laguerre polynomials $L_{n}^{(\alpha)}(x ; c)$ studied in [2]:

$$
B_{0} y^{\prime \prime \prime \prime}+B_{1} y^{\prime \prime \prime}+B_{2} y^{\prime \prime}+B_{3} y^{\prime}+B_{4} y=0
$$

where

$$
\begin{gathered}
B_{0}=x^{2}, \quad B_{1}=5 x, \quad B_{2}=-x^{2}+2 x(n+\alpha+2 c)-\alpha^{2}+4, \\
B_{3}=3[-x+(n+\alpha+2 c)], \quad B_{4}=n(n+2) .
\end{gathered}
$$

This rather nice equation was not given in the reference [2]. To construct a basis of solutions of this equation, we use the facts

$$
\begin{aligned}
\lim _{\beta \rightarrow \infty} \widehat{P}_{n+c}^{(\alpha, \beta)}(1-x / \beta)= & \left(\begin{array}{c}
\alpha+n+c \\
n+c
\end{array}\right) \Phi(-n-c, 1+\alpha ; x), \\
\lim _{\beta \rightarrow \infty} \widehat{Q}_{n+c}^{(\alpha, \beta)}(1-x / \beta)=- & \frac{\pi}{2} \csc (\alpha \pi)\left(\begin{array}{c}
\alpha+n+c \\
n+c
\end{array}\right) \Phi(-n-c, 1+\alpha ; x) \\
& \quad+\frac{\cos (\alpha \pi)}{2} \Gamma(\alpha) x^{-\alpha} \Phi(-n-c-\alpha, 1-\alpha ;-x) .
\end{aligned}
$$

$\Phi$ is the standard notation for the confluent hypergeometric function ${ }_{1} F_{1}$ [7]. Rather than employing the above functions, it is more convenient to construct a basis of solutions of the equation (3.20) from the functions

$$
\begin{array}{ll}
s_{1}=e^{-x} \Phi(1-c-\alpha, 1-\alpha ; x), & s_{2}=x^{\alpha} e^{-x} \Phi(1-c, 1+\alpha ; x), \\
t_{1}=\Phi(-n-c, 1+\alpha ; x), & t_{2}=x^{-\alpha} \Phi(-n-c-\alpha, 1-\alpha ; x) .
\end{array}
$$

A basis for the differential equation (3.20) is

$$
s_{i} t_{j}, \quad i, j=1,2 .
$$

Note that we must exclude those cases when $\alpha$ is an integer since in those cases neither the set $s_{1}, s_{2}$ nor the set $t_{1}, t_{2}$ will be linearly independent. In such cases, it is better to use the Tricomi $\Psi$ function to construct the solutions $s_{2}$ and $t_{2}$. We do not go into the details of this procedure, which is straightforward.

Again, the case $c=1$ is of particular interest. The basis of (3.20) becomes

$$
\begin{gathered}
x^{\alpha} e^{-x} L_{n+1}^{(\alpha)}(x), \quad e^{-x} \Phi(-\alpha, 1-\alpha ; x) L_{n+1}^{(\alpha)}(x), \\
e^{-x} \Phi(-n-\alpha-1,1-\alpha ; x), \\
e^{-x} x^{-\alpha} \Phi(-\alpha, 1-\alpha ; x) \Phi(-n-\alpha-1,1-\alpha ; x) .
\end{gathered}
$$


As with the associated Jacobi polynomials, ratios of these polynomials occur as Padé approximants,

$$
\begin{aligned}
\frac{L_{n}^{(\alpha)}(-x ; c+1)}{L_{n+1}^{(\alpha)}(-x ; c)}=\frac{(c+1) \Psi(c+\alpha+1, \alpha+1 ; x)}{\Psi(c+\alpha, \alpha+1 ; x)}+O\left(e^{-4 \sqrt{n x}}\right), & \\
& n \rightarrow \infty, \quad|\arg x|<\pi .
\end{aligned}
$$

The left-hand side is the $[n / n+1]$ Padé approximant about $\infty$ to the ratio on the right.

\section{Associated polynomials}

Let $\left\{p_{n}(x)\right\}$ be a family of orthonormal polynomials generated by (1.3) and (1.4). Assume further that the coefficients $a_{n}$ and $b_{n}$ are defined when $n$ is a continuous variable in $[0, \infty)$, as it is the case, for example, when $a_{n}$ and $b_{n}$ are rational functions of $n$ or of $q^{n}$. The associated polynomials $\left\{p_{n}^{(c)}(x)\right\}$ of order $c, c \geq 0$, of $p_{n}(x)$ are orthogonal polynomials satisfying

$$
x p_{n}^{(c)}(x)=a_{n+c+1} p_{n+1}^{(c)}(x)+b_{n+c} p_{n}^{(c)}(x)+a_{n+c} p_{n-1}^{(c)}(x), \quad n>0,
$$

and given initially by

$$
p_{0}^{(c)}(x):=1, \quad p_{1}^{(c)}(x)=\left(x-b_{c}\right) / a_{c+1} .
$$

The polynomials $p_{n-1}^{(1)}(x)$ are multiples of the numerator polynomials $\left\{p_{n}^{*}(x)\right\}$ where

$$
p_{0}^{*}(x)=0, \quad p_{1}^{*}(x)=1 / a_{1} .
$$

Recall that the functions of the second kind, $Q_{n}(z)$ corresponding to the polynomials $p_{n}(z)$ of (1.1) and (1.3), are defined as [20, 10]

$$
Q_{n}(z)=\frac{1}{w(z)} \int_{a}^{b} \frac{p_{n}(t)}{z-t} w(t) d t
$$

Strictly speaking, on one hand, $z$ must be off the cut $[a, b]$ for the function $Q_{n}(z)$ to be defined by the integral in (4.4). On the other hand, $z$ must be on $(a, b)$ in order for $w(z)$ to be defined. However, it is relatively straightforward to define $Q_{n}(z)$ for $x \in(a, b)$ using a Cauchy principal value integral. Thus, in what follows, we may assume $z=x$ is real. The $Q_{n}$ s satisfy both the differential equation (1.8) and the recurrence relation (1.3); see [10]. Throughout the rest of this section we shall assume

$$
c=1,2, \ldots
$$

Since $p_{n+c}(x)$ and $Q_{n+c}(x)$ form a basis of solutions of the second-order difference equation

$$
x y_{n}(x)=a_{n+c+1} y_{n+1}(x)+b_{n+c} y_{n}(x)+a_{n+c} y_{n-1}(x),
$$

and $p_{n}^{(c)}(x)$ also satisfies (4.6), then $p_{n}^{(c)}(x)$ must be a linear combination of $p_{n+c}(x)$, $Q_{n+c}(x)$. For $c \geq 1$, the initial conditions (4.2) imply

$$
p_{n}^{(c)}(x)=\frac{Q_{c-1}(x) p_{n+c}(x)-p_{c-1}(x) Q_{n+c}(x)}{Q_{c-1}(x) p_{c}(x)-p_{c-1}(x) Q_{c}(x)} .
$$


From the discrete analogue of the Wronskian, [1, 19]

$$
a_{n+1}\left[p_{n+1}(x) Q_{n}(x)-Q_{n+1}(x) p_{n}(x)\right]=a_{k+1}\left[p_{k+1}(x) Q_{k}(x)-Q_{k+1}(x) p_{k}(x)\right]
$$

which holds for $n \geq k \geq 0$, the initial conditions (1.4), and the integral definition (4.4), we get

$$
\begin{aligned}
a_{c}\left[Q_{c-1}(z) p_{c}(z)-p_{c-1}(z) Q_{c}(z)\right] & =a_{1} p_{1}(z) Q_{0}(z)-a_{1} Q_{1}(z) \\
& =\left(z-b_{0}\right) Q_{0}(z)-a_{1} Q_{1}(z) \\
& =\frac{1}{w(z)} \int_{a}^{b} \frac{z-b_{0}-a_{1} p_{1}(y)}{z-y} w(y) d y .
\end{aligned}
$$

Thus,

$$
a_{c}\left[Q_{c-1}(z) p_{c}(z)-p_{c-1}(z) Q_{c}(z)\right]=\frac{1}{w(z)}
$$

This establishes the relationship

$$
\frac{p_{n}^{(c)}(z)}{w(z)}=a_{c}\left[Q_{c-1}(z) p_{n+c}(z)-p_{c-1}(z) Q_{n+c}(z)\right] .
$$

Observe that (4.9) is the general form of (3.6), while (4.10) is the general version of (3.4).

From (1.8), $p_{n+c}(x), Q_{n+c}(x)$ satisfy the differential equation

$$
u^{\prime \prime}(x)+R_{n+c}(x) u^{\prime}(x)+S_{n+c}(x) u(x)=0,
$$

and $p_{c-1}(x), Q_{c-1}(x)$ satisfy the differential equation

$$
v^{\prime \prime}(x)+R_{c-1}(x) v^{\prime}(x)+S_{n-1}(x) v(x)=0 .
$$

The product of a solution of (4.11) and a solution of (4.12) will satisfy a fourthorder linear homogeneous differential equation, which may be found by the method explained in Section 3. The derivation is straightforward, and the equation results upon expanding the determinant

$$
\left|\begin{array}{ccccc}
y & a_{1} & a_{2} & a_{3} & a_{4} \\
y^{\prime} & b_{1} & b_{2} & b_{3} & b_{4} \\
y^{\prime \prime} & c_{1} & c_{2} & c_{3} & c_{4} \\
y^{\prime \prime \prime} & d_{1} & d_{2} & d_{3} & d_{4} \\
y^{\prime \prime \prime \prime} & e_{1} & e_{2} & e_{3} & e_{4}
\end{array}\right|=0
$$


with the coefficients $a_{j}, b_{j}, c_{j}, d_{j}, e_{j}, 1 \leq j \leq 4$ given by

$$
\begin{aligned}
& a_{1}=1, \quad a_{2}=0, \quad a_{3}=0, \quad a_{4}=0, \\
& b_{1}=0, \quad b_{2}=1, \quad b_{3}=1, \quad b_{4}=0, \\
& c_{1}=-S-s, \quad c_{2}=-r, \quad c_{3}=-R, \quad c_{4}=2, \\
& d_{1}=-s^{\prime}-S^{\prime}+R S+r s, \quad d_{2}=-s-r^{\prime}-3 S+r^{2}, \\
& d_{3}=-S-R^{\prime}-3 s+R^{2}, \quad d_{4}=-3 R-3 r, \\
& e_{1}=2 R^{\prime} S+R S^{\prime}+r s^{\prime}+2 r^{\prime} s-r^{2} s+6 s S-R^{2} S-s^{\prime \prime}-S^{\prime \prime}+s^{2}+S^{2}, \\
& e_{2}=-4 S^{\prime}+2 r s+4 R S+6 r S+3 r r^{\prime}-2 s^{\prime}-r^{3}-r^{\prime \prime}, \\
& e_{3}=-4 s^{\prime}+2 R S+4 r s+6 R s+3 R R^{\prime}-2 S^{\prime}-R^{3}-R^{\prime \prime}, \\
& e_{4}=-4 R^{\prime}-4 S-4 s+4 r^{2}+6 r R .
\end{aligned}
$$

In the above

$$
r:=R_{c-1}(x), \quad s:=S_{c-1}(x), \quad R:=R_{n+c}(x), \quad S:=S_{n+c}(x) .
$$

Obviously,

$$
p_{n}^{(c)}(x) / w(x)
$$

is a solution of the differential equation (4.13). A full basis of solutions of this equation is

$$
\left\{p_{n+c}(x) p_{c-1}(x), Q_{n+c}(x) p_{c-1}(x), p_{n+c}(x) Q_{c-1}(x), Q_{n+c}(x) Q_{c-1}(x)\right\} .
$$

The differential equation for the associated polynomials $p_{n}^{(c)}(x)$ can be found from (4.13) by a straightforward change of variable, but this equation will generally be more complicated than (4.13). Recall that if $v$ is a polynomial and $b=-a=\infty$, as is the case for the Freud weights, then $A_{n}$ and $B_{n}$ are polynomials. On the other hand, if $v$ is a rational function, then $A_{n}$ and $B_{n}$ are also rational functions. In either case, the $R_{n}$ s and $S_{n}$ s of (1.8)-(1.10) are rational functions, hence the differential equation (4.3) can be written as a fourth-order differential equation with polynomial coefficients.

\section{Co-recursive polynomials}

The initial conditions (1.4) and the recurrence relation (1.3) essentially amount to defining $a_{0} p_{-1}(x)$ as zero. In many cases, $a_{n}$ is defined through a pattern which makes $a_{0} \neq 0$. Chihara [6] introduced the concept of co-recursive orthogonal polynomials, where the second initial condition in (1.4) is changed to make $a_{0} p_{-1}(x) \neq 0$. Basically, this leads to a second linearly independent solution of (1.3). Some orthogonal polynomials that come from certain birth and death processes naturally lead to two different choices for $p_{1}(x)$ (or $p_{-1}(x)$ ), as pointed out in [11]. This observation also leads to the concept of zero balanced solutions of recurrence relations defined in [12].

In the case of associated orthogonal polynomials generated by (4.1), we may generate a second family of orthogonal polynomials $\left\{q_{n}^{(c)}(x)\right\}$ by demanding that it satisfies (4.1) for $n \geq 0$ and is given initially by

$$
q_{-1}^{(c)}(x)=\alpha x+\beta, \quad q_{0}^{(c)}(x)=1
$$


so that

$$
a_{c+1} q_{1}^{(c)}(x)=x-b_{c}-a_{c}(\alpha x+\beta) .
$$

With the initial conditions (5.1), it easily follows that

$$
q_{n}^{(c)}(x)=p_{n}^{(c)}(x)-\frac{a_{c}}{a_{c+1}} q_{-1}^{(c)}(x) p_{n-1}^{(c+1)}(x) .
$$

Combining (5.3) and (4.10), we arrive at the representation

$$
\frac{q_{n}^{(c)}(z)}{a_{c} w(z)}=\left[Q_{c-1}(z)-q_{-1}^{(c)}(x) Q_{c}(z)\right] p_{n+c}(z)-\left[p_{c-1}(z)-q_{-1}^{(c)}(x) p_{c}(z)\right] Q_{n+c}(z)
$$

Now find the first four derivatives of (5.4) and use the fact that both $p_{n+c}$ and $Q_{n+c}$ satisfy differential equation (1.8), with $n$ replaced by $n+c$, to obtain five equations in the four unknowns $p_{n+c}(x), p_{n+c}^{\prime}(x), Q_{n+c}(x)$, and $Q_{n+c}^{\prime}(x)$. Eliminating the unknowns $p_{n+c}(x), Q_{n+c}(x)$, and their first derivatives leads to a fourth-order differential equation for $q_{n}^{(c)}(z) / w(z)$.

It must be noted that the differential equation for the left-hand side of (5.4) which will result from the above analysis suffers from the fact that its coefficients are transcendental functions, rather than rational functions of $z$. A differential equation with rational function coefficients can be derived but will have a much higher order. Let $u, v$, and $w$ satisfy different linear homogeneous second-order differential equations with rational function coefficients. Then it is easily seen that $u+v$ satisfies a fourthorder equation of the same kind, and $(u+v) w$ satisfies an eighth-order equation of the same kind. Thus, the left-hand side of (5.4) will satisfy an eighth-order equation with rational coefficients.

\section{Remarks}

A referee kindly pointed out that Grosjean $[8,9]$ derived a fourth-order differential equation for the associated Legendre polynomials and special associated Jacobi polynomials with integer association parameter. As already mentioned in the introduction, Wimp [21] derived the fourth order differential equation for associated Jacobi polynomials with general nonnegative association parameter. In fact, Wimp [21] also found the weight function for the general associated Jacobi polynomials. Since all classical orthogonal polynomials are either special or limiting cases of Jacobi polynomials, Wimp's work also covers all associated Jacobi classical orthogonal polynomials. One referee reminded us of the references $[14,15,16,18]$ on co-recursive polynomials which contain differential equations for very special cases of co-recursive classical orthogonal polynomials.

We thank both referees for their constructive criticisms and helpful remarks.

Acknowledgment. Research partially supported by NSF grants DMS-9625459 and DMS-9625341.

\section{References}

1. N. I. Akhiezer, The Classical Moment Problem and Some Related Questions in Analysis, English translation, Oliver and Boyd, Edinburgh, 1965.

2. R. Askey and J. Wimp, Associated Laguerre and Hermite Polynomials, Proc. Royal Society Edinburgh, 96-A (1984), 15-37. 
3. W. Bauldry, Estimates of asymmetric Freud polynomials on the real line, J. Approximation Theory 63 (1990), 225-237.

4. S. S. Bonan and D. S. Clark, Estimates of the Hermite and the Freud polynomials, J. Approximation Theory 63 (1990), 210-224.

5. Y. Chen and M. E. H. Ismail, Ladder operators and differential equations for orthogonal polynomials, J. Phys. A 30 (1997), 7818-7829.

6. T. S. Chihara, On co-recursive orthogonal polynomials, Proceedings Amer. Math. Soc. 8 (1957), 899-905.

7. A. Erdélyi, W. Magnus, F. Oberhettinger, and G. F. Tricomi, Higher Transcendental Functions, Vol. 2, McGraw-Hill, New York, 1953.

8. C. C. Grosjean, Theory of recursive generation of systems of orthogonal polynomials: An illustrative example, J. Comp. Appl. Math. 12,13 (1985), 299-318.

9. orthogonal polynomials of the second kind associated with Jacobi and the Gegenbauer polynomials, J. Comp. Appl. Math. 16 (1985), 259-307.

10. M. E. H. Ismail, On discriminants and functions of the second kind of orthogonal polynomials, Results in Mathematics 34 (1998), 132-149.

11. M. E. H. Ismail, J. Letessier, D. Masson, and G. Valent, Birth and death processes and orthogonal polynomials. In: Orthogonal Polynomials: Theory and Practice, (P. Nevai, ed.), Proc. NATO ASI on Orthogonal Polynomials and Their Applications, Kluwer Academic Publishers, Dordrecht, 1990, pp. 225-229.

12. M. E. H. Ismail, J. Letessier, G. Valent, and J. Wimp, Two families of associated Wilson polynomials, Canadian J. Math. 42 (1990), 659-695.

13. M. E. H. Ismail and M. Rahman, Associated Askey-Wilson polynomials, Transactions Amer. Math. Soc. 328 (1991), 201-239.

14. J. Letessier, Co-recursive associated Laguerre polynomials, J. Comp. Appl. Math 49 (1993), $127-136$.

15. S_ Some results on co-recursive associated Laguerre and Jacobi polynomials, SIAM J. Math. Anal. 25 (1994), 528-548.

16. __ On co-recursive associated Jacobi polynomials, J. Comp. Appl. Math 57 (1995), 202-213.

17. E. D. Rainville, Special Functions, Chelsea, Bronx, 1971.

18. A. Ronveaux and F. Marcellan, Co-recursive orthogonal polynomials and fourth order equations, J. Comp. Appl. Math. 25 (1989), 105-109.

19. J. Shohat and J. D. Tamarkin, The Problem of Moments, revised edition, American Mathematical Society, Providence, 1950.

20. G. Szegő, Orthogonal Polynomials, Fourth Edition, American Mathematical Society, Providence, 1975.

21. J. Wimp, Explicit formulas for the associated Jacobi polynomials, Canadian J. Math. 39 (1987), 893-1000.

Department of Mathematics, University of South Florida, Tampa, Florida 33620-5700

Department of Mathematics and Computer Science, Drexel University, Philadelphia, PennSYLVANIA $19104-2884$ 